\title{
PERSISTÊNCIA DE AGROTÓXIC̃̃̃ UTILIZADOS NA PRODUÇÃo INTEGRADA DE MAÇÃ A Trichogramma pretiosum
}

\author{
Persistence of pesticides used in integrated production of apple to Trichogramma pretiosum
}

\author{
Sandro Daniel Nörnberg1, Anderson Dionei Grützmacher², Adalécio Kovaleski, \\ Jonas Alex Finatto ${ }^{2}$, Murilo Damé Fonseca Paschoal ${ }^{2}$
}

\begin{abstract}
RESUMO
No sistema de Produção Integrada de Maçã (PIM) o controle biológico de pragas é uma importante tática de controle, no entanto, a sua utilização pode ser limitada pela utilização de agrotóxicos. Nesse contexto, objetivou-se, com este trabalho, avaliar a persistência (duração da atividade nociva) de dez agrotóxicos recomendados na PIM sobre o parasitóide de ovos Trichogramma pretiosum. Insetos adultos foram expostos ao contato com resíduos de agrotóxicos pulverizados sobre folhas de videira cv. Isabel, sendo avaliado o efeito residual dos agrotóxicos sobre o parasitismo de T. pretiosum, aos 3,10,17, 24 e 31 dias após a pulverização, de acordo com a metodologia sugerida pela IOBC. O parâmetro utilizado para classificar os agrotóxicos foi a redução no parasitismo. Os resultados demonstraram que os produtos comerciais/ingredientes ativos ( $\mathrm{g}$ ou mL da formulação comercial.100 $\mathrm{L}^{-1}$ ): $\mathrm{o}$ inseticida Malathion ${ }^{\circledR} 1000 \mathrm{CE} /$ malationa (100) e os fungicidas Domark $^{\circledR} 100 \mathrm{CE} /$ tetraconazole (50), Manzate ${ }^{\circledR} 800 /$ mancozebe (200), Mancozebe Sipcam ${ }^{\otimes} /$ mancozebe (200) e Metiltiofan $^{\circledR} /$ tiofanato metilico (90) foram classificados como de vida curta $(<5$ dias de ação nociva); o inseticida/acaricida Vertimec ${ }^{\circledR} 18 \mathrm{CE} /$ abamectina (100) como levemente persistente (5-15 dias de ação nociva), o inseticida Lorsban ${ }^{\circledR} 480 \mathrm{BR} /$ clorpirifós (150) como moderadamente persistente (16-30 dias de ação nociva) e os inseticidas Imidan ${ }^{\circledR} 500$ PM/fosmete (200) e Sevin ${ }^{\circledR} 480$ SC/carbaril (360) e o fungicida/acaricida Kumulus ${ }^{\circledR}$ DF/enxofre (600) como persistentes (> 31 dias de ação nociva) a T. pretiosum.
\end{abstract}

Termos para indexação: Acaricidas, efeito residual, parasitóide de ovos, fungicidas, inseticidas, seletividade.

\section{ABSTRACT}

In the Integrated Production of Apple (IPA) the biological control is an important control tactics, however, it may be limited by the use of pesticides. Thus, the objective of this work was to evaluate the persistence (duration of harmful activity) of ten pesticides indicated in the IPA on the egg parasitoid Trichogramma pretiosum. Insect adults were exposed to pesticide residues on the leaves of vine cv. Isabel. The residue effect was evaluated on parasitism of T. pretiosum, at 3, 10, 17, 24 and 31 days after the treatment, using the IOBC standard methodology. The reduction in capacity of parasitism of T. pretiosum was used to measure the effect of the pesticides. The results showed that the products, commercial name/active ingredient ( $\mathrm{g}$ or $\mathrm{mL}$ commercial

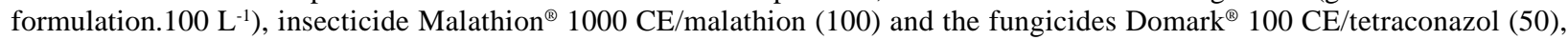
Manzate ${ }^{\circledR} 800$ /mancozeb (200), Mancozeb Sipcam ${ }^{\circledR} /$ mancozeb (200) and Metiltiofan $^{\circledR} /$ thiophanate-methyl (90) were classified as short lived (<5 days); the insecticide/acaricid Vertimec ${ }^{\circledR} 18 \mathrm{CE} /$ abamectin $(100)$ as slightly persistent (5-15 days of harmful action), the insecticide Lorsban ${ }^{\circledR} 480 \mathrm{BR} /$ chlorpyrifos (150) as moderately persistent (16-30 days of harmful action), the insecticides Imidan ${ }^{\circledR} 500 \mathrm{PM} /$ phosmet (200) and Sevin ${ }^{\circledR} 480 \mathrm{SC} /$ carbaryl (360) and the fungicide/acaricid Kumulus ${ }^{\circledR}$ DF/sulphur (600) were persistent (> 31 days of harmful action) to T. pretiosum.

Index terms: Acaricides, residue effect, egg parasitoids, fungicides, insecticides, selectivity.

(Recebido em 3 de agosto de 2009 e aprovado em 28 de maio de 2010)

\section{INTRODUÇÃo}

A cultura da macieira, através da implementação do sistema de Produção Integrada de Maçã (PIM), foi a primeira frutífera com certificação no Brasil. A adequação da cultura da macieira às normas desse sistema é uma exigência do mercado internacional, principalmente da União Européia que, desde 2003, restringe a importação de frutas quando produzidas no sistema convencional (Kovaleski, 2004).

Com a implantação da PIM, algumas pragas consideradas primárias passaram a ser secundárias, tendo como exemplo o ácaro vermelho europeu Panonychus ulmi (Prostigmata: Tetranychidae), que até o início da década de 90 era a principal praga da macieira, sendo atualmente

\footnotetext{
${ }^{1}$ Universidade Federal de Pelotas/UFPEL - Faculdade de Agronomia Eliseu Maciel/FAEM - Departamento de Fitossanidade/DFS - Laboratório de Manejo Integrado de Pragas/LabMip - Campus Universitário - Cx. P. 354 - 96010-900 - Capão do Leão, RS - sandro_ufpel@hotmail.com

${ }^{2}$ Universidade Federal de Pelotas/UFPEL - Faculdade de Agronomia Eliseu Maciel/FAEM - Departamento de Fitossanidade/DFS - Capão do Leão, RS ${ }^{3}$ Empresa Brasileira de Pesquisa Agropecuária/Embrapa - Centro Nacional de Pesquisa de Uva e Vinho - Estação Experimental de Fruticultura Temperada - Vacaria, RS
} 
considerada secundária. Provavelmente, isso ocorreu devido aos avanços no manejo integrado de pragas (MIP) associado à restrição na utilização de produtos de largo espectro, como os piretróides e alguns fosforados, favorecendo o controle biológico natural desta praga.

No entanto, outras pragas, até então de importância secundária na cultura da macieira, começaram a ocorrer com maior intensidade, como a mariposa oriental, Grapholita molesta (Busck, 1916) (Lepidoptera: Tortricidae) (Kovaleski, 2007) e as chamadas "grandes lagartas", das famílias Noctuidae e Geometridae (Fonseca, 2006), isso provavelmente devido ao monitoramento das pragas-chave e consequente redução no uso de agrotóxicos.

Assim, fica evidente o dinamismo de um agroecossistema, em que novos métodos de controle são eficientes para determinada praga e ao mesmo tempo propiciam condições para o desenvolvimento de outras pragas. Nesse contexto, o MIP torna-se indispensável, sendo o controle biológico de pragas uma ferramenta fundamental, seja de forma natural ou aplicada nos pomares de macieira no Sul do Brasil (Kovaleski, 2004; Monteiro \& Souza, 2004).

Dentre os inimigos naturais com potencial para o controle de insetos-praga na cultura da macieira, destacamse os parasitóides de ovos do gênero Trichogramma. A espécie Trichogramma pretiosum Riley, 1879 (Hymenoptera: Trichogrammatidae) apresenta potencial no parasitismo de ovos de G. molesta (Pinto et al., 2002) e Bonagota salubricola (Meyrick, 1937) (Lepidoptera: Tortricidae) (Monteiro et al., 2004), demonstrando a sua importância como agente biológico em um programa de controle biológico aplicado.

No entanto, a utilização desses parasitóides, na cultura da macieira, ainda apresenta várias limitações, sendo a carência de informações sobre a seletividade dos agrotóxicos utilizados sobre organismos benéficos, uma delas. Manzoni et al. (2006a,b,c) avaliaram a seletividade de alguns agrotóxicos, recomendados na PIM, sobre T. pretiosum em condições de laboratório e recomendaram a realização de estudos de persistência biológica para aqueles agrotóxicos considerados nocivos aos adultos do parasitóide. Entretanto, para a cultura da macieira, ainda inexistem trabalhos de persistência biológica com parasitóides de ovos, necessitando assim, estudos para avaliar o comportamento dos agrotóxicos em condições de semicampo e campo.

Em estudos realizados em laboratório, o inimigo natural geralmente é submetido à máxima exposição dos resíduos dos produtos. Assim, os compostos que se revelaram tóxicos para $T$. pretiosum em laboratório, podem ter seus efeitos reduzidos quando aplicados em condições de casa-de-vegetação e campo, sendo uma das razões a degradação dos compostos (Silva \& Fay, 2004).

Para a cultura do pessegueiro, Grützmacher et al. (2004) realizaram bioensaios em laboratório e de persistência biológica com agrotóxicos recomendados na Produção Integrada de Pêssego (PIP) sobre a espéciepadrão da IOBC/WPRS, Trichogramma cacoeciae (Hymenoptera: Trichogrammatidae); posteriormente, Giolo et al. (2008) avaliaram a seletividade de agrotóxicos recomendados na PIP, com testes de persistência, sobre T. pretiosum. Em ambos os trabalhos, foi observado que muitos produtos considerados tóxicos em laboratório foram pouco persistentes em condições de casa-de-vegetação, reiterando, dessa forma, a importância do prosseguimento dos testes de seletividade.

Neste contexto, objetivou-se, com o presente estudo, avaliar a persistência (duração da atividade nociva) de agrotóxicos (acaricidas, fungicidas e inseticidas) indicados na PIM sobre adultos de $T$. pretiosum em condições de casa-de-vegetação.

\section{MATERIAL E MÉTODOS}

Os bioensaios foram realizados seguindo metodologias padronizadas pela "International Organization for Biological and Integrated Control of Noxious Animals and Plants, West Palaearctic Regional Section" (IOBC/ WPRS) (Hassan \& Abdelgader, 2001), sendo conduzidos em laboratórios e casa-de-vegetação do Departamento de Fitossanidade (DFs) da Faculdade de Agronomia "Eliseu Maciel" (FAEM) na Universidade Federal de Pelotas (UFPel). O material biológico foi constituído por parasitóides de ovos da espécie $T$. pretiosum oriundos de criação de laboratório, em câmaras climatizadas (temperatura $25 \pm 1^{\circ} \mathrm{C}$, umidade relativa $70 \pm 10 \%$ e fotofase 14 horas) utilizando-se ovos do hospedeiro alternativo Anagasta kuehniella (Zeller, 1879) (Lepidoptera: Pyralidae).

Devido à capacidade operacional do sistema instalado no laboratório (seis tratamentos por bioensaio), os estudos de seletividade foram divididos em dois grupos, inseticidas/acaricidas e fungicidas/acaricidas, sendo que cada grupo de agrotóxicos foi conduzido durante cinco semanas, iniciando-se cada bioensaio aos 3, 10, 17, 24 e 31 dias após a pulverização, correspondendo a um bioensaio por semana.

Os produtos-teste estão registrados e indicados para uso na PIM e foram avaliados nas dosagens máximas indicadas pelos fabricantes (Tabela 1). O tratamento testemunha foi constituído somente por água destilada. 
Tabela 1 - Agrotóxicos avaliados nos testes residuais para Trichogramma pretiosum, empregando-se dosagem máxima do produto formulado utilizado na Produção Integrada de Maçã (PIM).

\begin{tabular}{lllcccc}
\hline \multicolumn{1}{c}{ Produto comercial } & \multicolumn{1}{c}{ Ingrediente ativo } & Grupo químico & Classe $^{(1)}$ & DC $^{(2)}$ & C.i.a. $^{(3)}$ & C.f.c. $^{(4)}$ \\
\hline Imidan $^{\circledR} 500 \mathrm{PM}$ & fosmete & Organofosforado & $\mathrm{I}$ & 200 & 0,100 & 0,20 \\
Lorsban $^{\circledR} 480 \mathrm{BR}$ & clorpirifós & Organofosforado & $\mathrm{I}$ & 150 & 0,072 & 0,15 \\
Malathion $^{\circledR} 1000 \mathrm{CE}$ & malationa & Organofosforado & $\mathrm{I}$ & 100 & 0,100 & 0,10 \\
Sevin $^{\circledR} 480 \mathrm{SC}$ & carbaril & Carbamato & $\mathrm{I}$ & 360 & 0,173 & 0,36 \\
Vertimec $^{\circledR} 18 \mathrm{CE}$ & abamectina & Avermectina & A/I & 100 & 0,002 & 0,10 \\
Domark $^{\circledR} 100 \mathrm{CE}$ & tetraconazole & Triazol & $\mathrm{F}$ & 50 & 0,005 & 0,05 \\
Kumulus $^{\circledR} \mathrm{DF}$ & enxofre & Inorgânico & F/A & 600 & 0,480 & 0,60 \\
Mancozebe Sipcam $^{\circledR}$ & mancozebe & Ditiocarbamato & $\mathrm{F}$ & 200 & 0,160 & 0,20 \\
Manzate $^{\circledR} 800$ & mancozebe & Ditiocarbamato & $\mathrm{F}$ & 200 & 0,160 & 0,20 \\
Metiltiofan $^{\circledR}$ & tiofanato metílico & Benzimidazol & $\mathrm{F}$ & 90 & 0,063 & 0,09 \\
\hline
\end{tabular}

${ }^{(1)} \mathrm{A}=$ acaricida, $\mathrm{F}=$ fungicida, $\mathrm{I}=$ inseticida; ${ }^{(2)} \mathrm{DC}=$ Dosagem da formulação comercial $\left(\mathrm{g}\right.$ ou mL.100 L $\left.{ }^{-1}\right) ;{ }^{(3)}$ C.i.a. = Concentração (\%) testada do ingrediente ativo na calda; ${ }^{(4)}$ C.f.c. = Concentração $(\%)$ testada da formulação comercial na calda.

Os testes de toxicidade foram conduzidos em laboratório $\left(25 \pm 1^{\circ} \mathrm{C}\right.$, umidade relativa $70 \pm 10 \%$ e fotofase de 14 horas), expondo-se adultos (estágio mais sensível) de $T$. pretiosum a resíduos secos de agrotóxicos (Tabela 1), contidos sobre folhas de videiras (planta padrão da IOBC/ WPRS) cv. Isabel, cultivadas em vasos plásticos e que receberam todos os tratos culturais necessários até alcançarem aproximadamente $80-100 \mathrm{~cm}$ de altura. A pulverização dos compostos foi realizada com pulverizador pressurizado a $\mathrm{CO}_{2}$ (pressão constante - 50 psi) até o ponto de escorrimento da calda. Após três horas desse procedimento, as plantas foram colocadas no interior da casa-de-vegetação.

Aos 3, 10, 17, 24 e 31 dias após a pulverização (DAP) dos compostos nas videiras, folhas de cada tratamento foram coletadas e expostas ao contato com adultos do parasitóide por meio de gaiolas de exposição, conforme Hassan \& Abdelgader (2001).

Para permitir o contato entre os insetos de T.pretiosum com as folhas tratadas, adultos com até 24 horas de idade foram inseridos nas gaiolas de exposição através de tubos de emergência (ampola de vidro transparente de $120 \mathrm{~mm}$ de comprimento por $20 \mathrm{~mm}$ de diâmetro em uma extremidade e $7 \mathrm{~mm}$ na outra), os quais foram desconectados após $16 \mathrm{~h}$ e utilizados no cálculo do número de parasitóides que entraram na gaiola (Hassan et al., 2000).

Para avaliar os efeitos dos compostos no parasitismo de $T$. pretiosum, foram ofertados cartões, contendo 3 círculos de $1 \mathrm{~cm}$ de diâmetro com $350 \pm 50$ ovos de A. kuehniella inviabilizados, às 24 (três cartões), 48 (dois cartões) e 96 (um cartão) horas após pulverização, para serem parasitados por fêmeas sobreviventes de
T. pretiosum para avaliação da capacidade de parasitismo, totalizando um período de cerca de 144 horas ( seis dias) em que ovos do hospedeiro alternativo ficaram disponíveis para o parasitismo.

Sete dias após o início de cada bioensaio, as gaiolas foram desmontadas e os cartões contendo ovos do hospedeiro alternativo, ofertados aos insetos em teste, foram acondicionados em placas de Petri descartáveis (90 x $15 \mathrm{~mm})$. Esses cartões foram acondicionados, nas mesmas condições do experimento, por mais três dias, permitindo o desenvolvimento do parasitóide até o estágio de pupa para realização da contagem do número de ovos parasitados.

O número de fêmeas em cada gaiola foi determinado pela seguinte fórmula: $F n=[(O n * I p)-I n] * r s$, em que $F n$ é o número de fêmeas na gaiola $n$; On é o número de ovos parasitados no círculo de $1 \mathrm{~cm}$ de diâmetro correspondente a repetição $n$; Ip é o número médio de parasitóides emergidos por ovo; In é o número de adultos que permaneceram no interior do tubo de emergência correspondente a repetição $n$; e $r s$ é a razão sexual da população em estudo. Os parâmetros, número médio de parasitóides por ovo e razão sexual, foram obtidos mediante avaliação de quatro círculos de $1 \mathrm{~cm}$ de diâmetro contendo ovos parasitados.

A redução no parasitismo causada pelos produtosteste, comparada com o tratamento testemunha, foi utilizada como parâmetro de classificação da toxicidade dos agrotóxicos em cada bioensaio (semana) conforme proposta da IOBC/WPRS. Posteriormente, baseado na classificação de toxicidade, os produtos foram classificados de acordo com a escala de persistência da IOBC/WPRS (Hassan \& Abdelgader, 2001) em: 1, vida curta 
(<5 dias); 2, levemente persistente (5-15 dias); 3, moderadamente persistente (16-30 dias) e 4 , persistente (>31 dias)

Cada gaiola de contato foi considerada uma unidade experimental. Os experimentos foram inteiramente casualizados e delineados com quatro repetições por tratamento. Análises estatísticas complementares foram realizadas com auxílio do programa estatístico SAS Statistical Analysis System (SAS Institute, 2002). Os resultados obtidos, quanto ao número de ovos parasitados por fêmea, foram testados quanto à normalidade pelo teste de Shapiro-Wilk através do procedimento UNIVARIATE. Não atendida essa pressuposição, foi realizada análise nãoparamétrica pelo procedimento npar1way (Kruskal-Wallis) e, após comprovação da existência de diferença entre tratamentos, os dados foram transformados pelo procedimento RANK. A comparação de médias foi obtida pelo teste Bonferroni-Dunn $t$ utilizando-se o procedimento GLM em nível de 5\% de probabilidade de erro. Em caso de normalidade dos dados, as médias foram comparadas pelo teste Tukey (dados balanceados) e Tukey-Kramer (dados não balanceados) utilizando-se o procedimento GLM em nível de $5 \%$.

\section{RESULTADOS E DISCUSSÃO}

Nos bioensaios com fungicidas/acaricidas, o número médio de ovos parasitados por fêmea de T. pretiosum nas testemunhas (água destilada) foram de 25,$43 ; 40,14 ; 25,76 ; 30,47$ e 25,31 e nos bioensaios com inseticidas/acaricidas foram de 25,86; 31,28; 33,42; 26,18 e 25,33 ovos parasitados por fêmea, correspondendo aos 3, 10, 17, 24 e 31 DAP, respectivamente (Tabela 2). Esses resultados dão credibilidade aos bioensaios do presente trabalho, uma vez que a metodologia padronizada pela IOBC/WPRS preconiza um número mínimo de 15 ovos parasitados por fêmea (Hassan et al., 2000).

Dos fungicidas/acaricidas avaliados, Domark ${ }^{\circledR} 100$ CE, Mancozebe Sipcam ${ }^{\circledR}$, Manzate ${ }^{\circledR} 800$ e Metiltiofan ${ }^{\circledR}$ reduziram a capacidade de parasitismo de $T$. pretiosum em mais de $30 \%$ somente até 3 DAP (Figura 1) e foram classificados como de vida curta (classe 1) (Tabela 2). No entanto Kumulus ${ }^{\circledR}$ DF apresentou efeito residual, aos 31 DAP, capaz de reduzir a capacidade de parasitismo de T. pretiosum em mais de $66,79 \%$ (Figura 1) e foi classificado como persistente (classe 4).

Resultados similares foram observados para T. cacoeciae com o fungicida Kumulus ${ }^{\circledR}$ DF (Grützmacher et al., 2004) e outro produto comercial à base de enxofre (Hassan, 1994; Hassan et al., 1998). No entanto, resultados diferentes foram verificados para o ingrediente ativo mancozeb, com diferentes formulações, classificando como levemente persistente (classe 2) (Hassan, 1994) e como persistente (classe 4) (Hassan, 1994; Hassan et al., 1998). Os resultados referentes aos fungicidas classificados como de vida curta (classe 1) podem ser explicados devido à falta de um sítio de ação específico dos fungicidas para o controle de insetos. No entanto, o produto Kumulus ${ }^{\circledR}$ DF (classe 4) além de ser um fungicida, também tem ação acaricida/inseticida e por isso possui um sítio de ação nos artrópodes, atuando nos processos metabólicos, na etapa final do processo respiratório, na cadeia de transporte de elétrons (Omoto, 2000), ou ainda pode estar associado à formulação ou produto "inerte" presente, visto que o

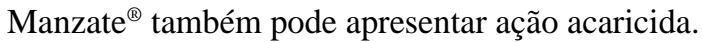

Dentre os inseticidas avaliados, Sevin ${ }^{\circledR} 480$ SC foi o mais tóxico, apresentando redução média na capacidade de parasitismo de $T$. pretiosum em mais de $99 \%$ aos 3, 10, 17, 24 e 31 DAP (Figura 2), sendo classificado como persistente (classe 4). Imidan ${ }^{\circledR} 500$ PM também foi classificado como persistente (classe 4), apresentando redução média de 72,86\% até os 31 DAP. Lorsban ${ }^{\circledR} 480$ BR foi classificado como moderadamente persistente (classe 3), apresentando uma redução média maior do que $30 \%$ até 17 DAP (Figura 2). O inseticida Malathion ${ }^{\circledR} 1000$ CE e o inseticida/acaricida Vertimec ${ }^{\circledR} 18 \mathrm{CE}$ foram classificados, respectivamente, como de vida curta (classe 1) e levemente persistente (classe 2) (Tabela 2).

A baixa persistência do Malathion ${ }^{\circledR} 1000 \mathrm{CE}$ e o Vertimec $^{\circledR} 18 \mathrm{CE}$, em condições de casa-de-vegetação, provavelmente pode ocorrer devido à degradação das estruturas moleculares desses inseticidas sob influência das condições climáticas. Resultados semelhantes de testes de persistência biológica, com adultos da espécie padrão da IOBC/WPRS T. cacoeciae, foram observados por Hassan et al. (1998) para Vertimec ${ }^{\circledR}$, classificando este inseticida/acaricida como levemente persistente (classe 2).

No entanto, resultados distintos foram encontrados para o ingrediente ativo malationa (Malathion $57 \mathrm{EC}$ ) por Youssef et al. (2004), em teste de persistência em folhas de oliveira, em que o classificaram como levemente persistente (classe 2). Essa variação nos resultados, possivelmente ocorreu devido às diferenças nas respostas fisiológicas das espécies $T$. pretiosum e $T$. cacoeciae a este inseticida, e, ou devido à maior dosagem do produto utilizado por esses autores (2,5 vezes à utilizada no presente trabalho) ou ainda devido ao substrato foliar utilizado, uma vez que a espécie de planta utilizada e as condições climáticas durante o período após aplicação (Wills \& McDowell, 1987) são os fatores que mais afetam a persistência de inseticidas em superfícies foliares. 


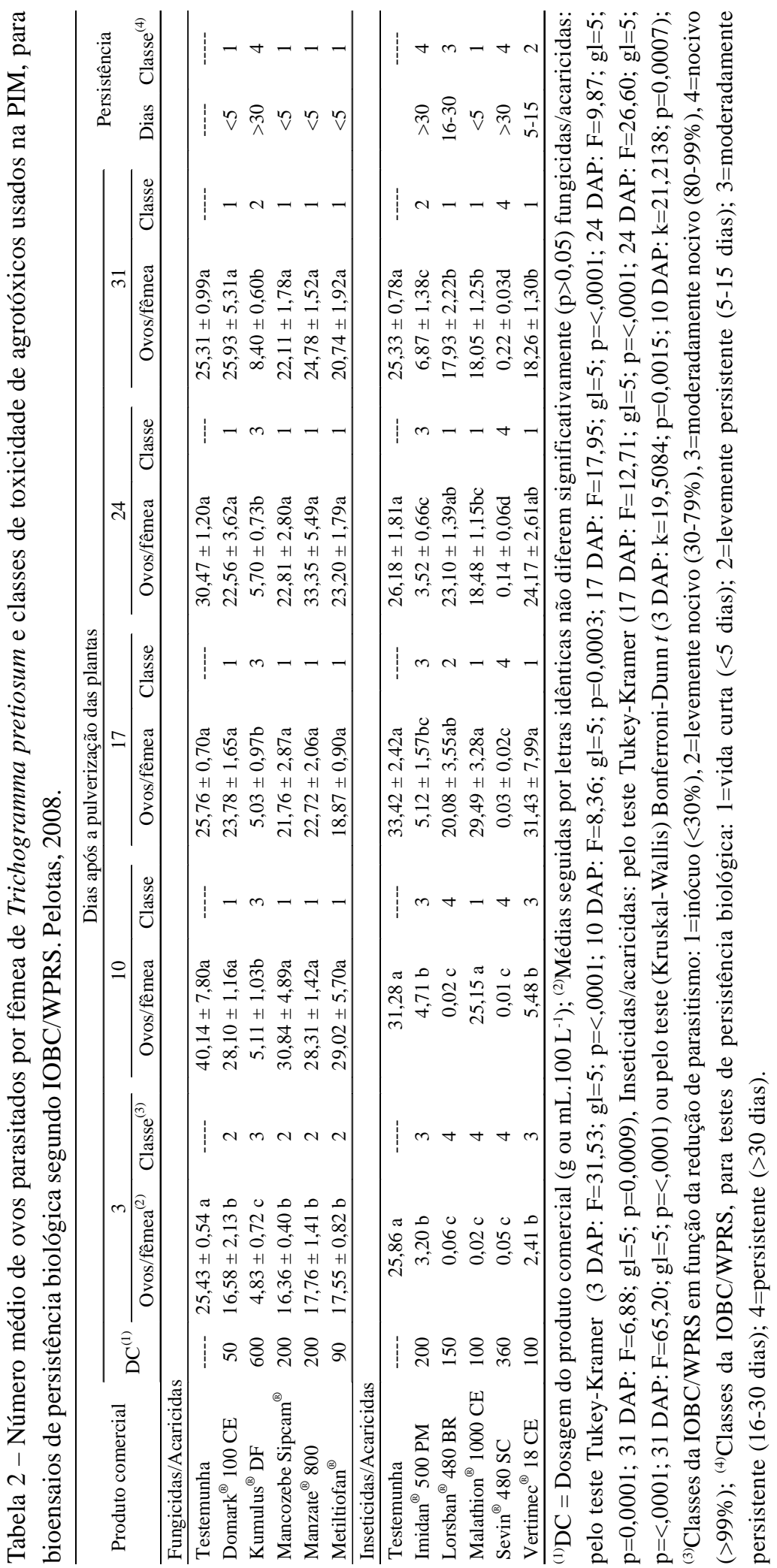

Ciênc. agrotec., Lavras, v. 35, n. 2, p. 305-313, mar./abr., 2011 


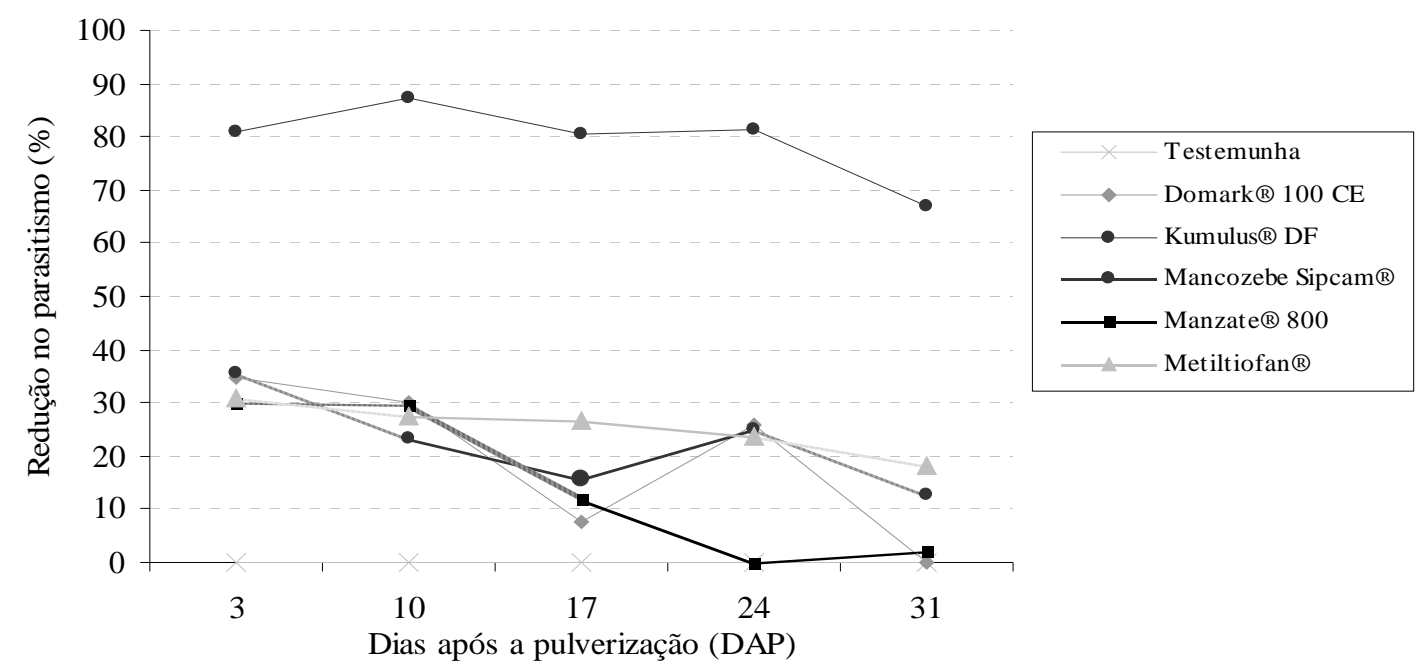

Figura 1 - Persistência de agrotóxicos, acaricidas e fungicidas, usados na PIM, sobre a capacidade de parasitismo de Trichogramma pretiosum em casa-de-vegetação. Pelotas, 2008.

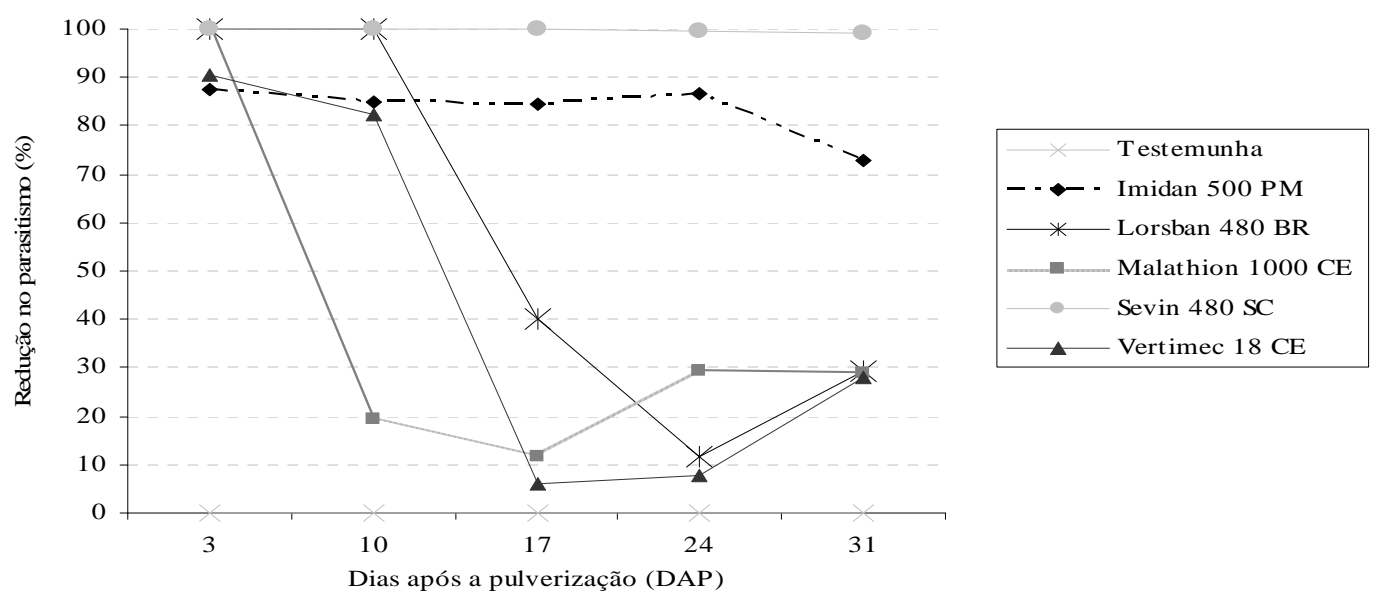

Figura 2 - Persistência de agrotóxicos, acaricidas e inseticidas, usados na PIM, sobre a capacidade de parasitismo de Trichogramma pretiosum em casa-de-vegetação. Pelotas, 2008.

Da mesma forma, resultados distintos foram verificados em testes de semicampo quando aplicaram o ingrediente ativo abamectina sobre plantas de tomateiro e avaliaram o efeito residual na capacidade de parasitismo de $T$. pretiosum, em que constataram que esse inseticida causou redução média de $86 \%$ somente 24 horas após a aplicação e, já aos 3 DAP, a redução na capacidade de parasitismo foi de aproximadamente $30 \%$ (Carvalho et al., 1999), portanto, diferindo do presente estudo.

Possíveis explicações para essa diferença nos resultados estão relacionadas às variações metodológicas que foram adotadas em cada trabalho. Além disso, diferenças de suscetibilidade intraespecíficas do parasitóide também podem estar relacionadas aos resultados obtidos em linhagens de $T$. pretiosum (Carvalho et al., 2001) e/ou características intrínsecas distintas entre as formulações comerciais dos agrotóxicos, pois os autores não mencionaram qual foi o produto comercial testado conforme recomenda a IOBC/WPRS (Hassan et al., 2000).

Os inseticidas Lorsban ${ }^{\circledR} 480$ BR, Imidan ${ }^{\circledR} 500$ PM e Sevin $^{\circledR} 480$ SC apresentaram elevada toxicidade em bioensaios em condições de laboratório, reduzindo a capacidade de parasitismo de T. pretiosum em 100\% (Hassan, 1994; Giolo et al., 2005). No presente estudo, mesmo sendo conduzido em 
casa-de-vegetação, Sevin ${ }^{\circledR} 480$ SC reduziu a capacidade de parasitismo em mais de $99 \%$ até $31 \mathrm{DAP}$, o que demonstra uma elevada persistência deste produto, sendo classificado como persistente (classe 4) (Tabela 2), o que foi verificado também em teste de persistência com $T$. cacoeciae com ingrediente ativo carbaril (Prosevor $\left.{ }^{\circledR}\right)$ (Hassan, 1994, 1998).

O inseticida Lorsban ${ }^{\circledR} 480$ BR diferentemente dos demais, apresentou elevada toxicidade somente até 10 DAP sendo dessa forma classificado como moderadamente persistente (classe 3). Esse comportamento pode ser devido a ação fumigante desse produto nos primeiros dias, uma vez que se pôde observar que, nos bioensaios aos 3 e 10 DAP, a maioria dos insetos adultos de $T$. pretiosum não entraram nas gaiolas de exposição, morrendo ainda dentro dos tubos de emergência logo após a conexão destes às gaiolas. Nos bioensaios posteriores, aos 17, 24 e 31 DAP, o número de insetos que entraram nas gaiolas foi semelhante aos demais tratamentos, e consequentemente houve a redução da ação tóxica deste inseticida sobre a capacidade de parasitismo dos insetos, o que provavelmente ocorreu devido à degradação das estruturas moleculares desse inseticida pela influência das condições climáticas. Diversos estudos têm sido realizados para avaliar a seletividade de clorpirifós para Trichogramma spp., sendo considerado como altamente tóxico para estes insetos, em todas suas fases (Hassan, 1994; Castelo Branco \& França, 1995; Brunner et al., 2001; Delpuech \& Meyet, 2003).

Brunner et al. (2001) concluíram que os inseticidas organofosforados e carbamatos apresentaram pequena ou nenhuma seletividade aos adultos de Trichogramma platneri Nagarkatti (Hymenoptera: Trichogrammatidae) em contato com os produtos pulverizados sobre folhas de macieira, corroborando com os resultados obtidos no presente estudo para os inseticidas Lorsban ${ }^{\circledR} 480 \mathrm{BR}$ (organofosforado), Imidan ${ }^{\circledR} 500$ PM (organofosforado) e Sevin $^{\circledR} 480$ SC (carbamato).

Testes de persistência biológica ajudam na estimativa da toxicidade de um agrotóxico, pois o seu impacto no campo é bastante afetado pela sua persistência. Agrotóxicos de vida curta (classe 1) poderiam ser utilizados com frequência em programas integrados de controle, na ausência de produtos inócuos. De acordo com a sequência de testes da IOBC/WPRS (Hassan \& Abdelgader, 2001) para Trichogramma spp., os agrotóxicos Lorsban ${ }^{\circledR} 480$ BR moderadamente persistente (classe 3), Imidan ${ }^{\circledR} 500$ PM, Sevin $^{\circledR} 480$ SC e Kumulus ${ }^{\circledR}$ DF que foram persistentes (classe 4) devem ser avaliados em condições de campo, sendo a última etapa de testes de seletividade, a qual poderá avaliar, de forma conclusiva, a toxicidade apresentada por esses agrotóxicos para T. pretiosum.

\section{CONCLUSÕES}

Para os produtos comerciais (g ou mL $100 \mathrm{~L}^{-1}$ ), conclui-se que: Malathion ${ }^{\circledR} 1000$ CE (100), Domark ${ }^{\circledR} 100$ CE (50), Manzate ${ }^{\circledR} 800$ (200), Mancozeb Sipcam ${ }^{\circledR}$ (200) e Metiltiofan $^{\circledR}(90)$ são de vida curta $(<5$ dias de ação nociva); Vertimec $^{\circledR} 18$ CE (100) é levemente persistente (5-15 dias de ação nociva); Lorsban ${ }^{\circledR} 480$ BR (150) moderadamente persistente (16-30 dias de ação nociva). Imidan ${ }^{\circledR} 500$ PM (200), Sevin ${ }^{\circledR} 480$ SC (360) e Kumulus ${ }^{\circledR}$ DF (600) são persistentes (> 31 dias de ação nociva).

\section{AGRADECIMENTOS}

Ao Conselho Nacional de Desenvolvimento Científico e Tecnológico (CNPq) e a Fundação de Amparo à Pesquisa do Estado do Rio Grande do Sul (FAPERGS) pelo apoio financeiro para a realização desta pesquisa.

\section{REFERÊNCIAS BIBLIOGRÁFICAS}

BRUNNER, J.F.; DUNLEY, J.E.; DOERR, M.D.; BEERS, E.H. Effect of pesticides on Colpoclypes florus (Hymenoptera: Eulophidae) and Trichogramma platneri (Hymenoptera: Trichogrammatidae), parasitoids of leafrollers in Washington. Journal of Economic Entomology, Lanham, v.94, p.1075-1084, 2001.

CARVALHO, G.A.; PARRA, J.R.P.; BAPTISTA, G.C. Ação residual de alguns inseticidas pulverizados em plantas de tomateiro sobre duas linhagens de Trichogramma pretiosum Riley, 1879 (Hymenoptera:

Trichogrammatidae) em casa-de-vegetação. Ciência e Agrotecnologia, Lavras, v.23, p.770-775, 1999.

CARVALHO, G.A.; PARRA, J.R.P.; BAPTISTA, G.C. Impacto de produtos fitossanitários utilizados na cultura do tomateiro na fase adulta de duas linhagens de Trichogramma pretiosum Riley, 1879 (Hymenoptera: Trichogramatidae). Ciência e Agrotecnologia, Lavras, v.25, p.560-568, 2001.

CASTELO BRANCO, M.; FRANÇA, F.H. Impacto de inseticidas sobre adultos de T. pretiosum. Horticultura Brasileira, Brasilia, v.13, p.199-201, 1995.

DELPUECH, J.M.; MEYET, J. Reduction in the sex ratio of the progeny of a parasitoid (Trichogramma brassicae) surviving the insecticide chlorpirifos. Archives of Environmental Contamination and Toxicology, New York, v.45, p.203-208, 2003. 
FONSECA, F.L. Ocorrência, monitoramento, caracterização de danos e parasitismo de Noctuidae e Geometridae em pomares de macieira em Vacaria, Rio Grande do Sul, Brasil. 2006. 97p. Tese (Doutorado em Entomologia) - Universidade federal do Paraná, CuritibaPR.

GIOLO, F.P.; GRÜTZMACHER, A.D.; MANZONI, C.G.; FACHINELLO, J.C.; GRÜTZMACHER, D.D.;

NÖRNBERG, S.D. Persistência de agrotóxicos indicados na Produção Integrada de Pêssego a Trichogramma pretiosum Riley, 1879 (Hymenoptera:

Trichogrammatidae). Revista Brasileira de Fruticultura, Jaboticabal, v.30, n.1, p.122-126, 2008.

GIOLO, F.P.; GRÜTZMACHER, A.D.; MANZONI, C.G.; FACHINELLO, J.C.; NÖRNBERG, S.D.; STEFANELLO JÚNIOR, G.J. Seletividade agrotóxicos indicados na produção integrada de pêssego a Trichogramma pretiosum (Hymenoptera: Trichogrammatidae). Revista Brasileira de Fruticultura, Jaboticabal, v.27, p.222-225, 2005.

GRÜTZMACHER, A.D.; ZIMMERMANN, O.; YOUSEF, A.; HASSAN, S.A. The side-effects of pesticides used in integrated production of peaches in Brazil on the egg parasitoid Trichogramma cacoeciae Marchal (Hymenoptera: Trichogrammatidae). Journal of Applied Entomology, Berlin, v.128, p.377-383, 2004.

HASSAN, S.A. Comparison of three different laboratory methods and one semi-field test method to assess the side effects of pesticides on Trichogramma cacoeciae Marchal (Hymenoptera: Trichogrammatidae). Bulletin IOBC/WPRS, Darmstadt, v.17, p.133-141, 1994.

HASSAN, S.A. The side effects of 161 pesticides on the egg parasitoid Trichogramma cacoeciae Marchal (Hymenoptera, Trichogrammatidae). Egg Parasitoids, Darmstadt, p.63-76, 1998.

HASSAN, S.A.; ABDELGADER, H. A sequential testing program to assess the effects of pesticides on Trichogramma cacoeciae Marchal (Hymenoptera: Trichogrammatidae). Pesticides and Beneficial Organisms: IOBC/WPRS Bulletin, Montfavet, v.24, p.71-81, 2001.

HASSAN, S.A.; HAFES, B.; DEGRANDE, P.E.; HERAI, $\mathrm{K}$. The side-effects of pesticides on the egg parasitoid
Trichogramma cacoeciae Marchal (Hymenoptera: Trichogrammatidae), acute dose-response and persistence tests. Journal of Applied Entomology, Berlin, v.122, n.9, p.569-573, 1998.

HASSAN, S.A.; HALSALL, N.; GRAY, A.P.; KUEHNER, C.; MOLL, M.; BAKKER, F.M.; ROEMBKE, J.; YOUSEF, A.; NASR, F.; ABDELGADER, H.A. A laboratory method to evaluate the side effects of plant protection products on Trichogramma cacoeciae Marchal (Hymenoptera: Trichogrammatidae). In: CANDOLFI, M.P.; BLÜMEL, S.; FORSTER, R.; BAKKER, F.M.;

KOVALESKI, A. Manejo de grafolita (Grapholita molesta) em macieira. Agapomi, Vacaria, n.163, p.6-8, 2007.

KOVALESKI, A. Pragas. In: __ Maçã: fitossanidade. Bento Gonçalves: Embrapa Uva e Vinho, 2004. p.10-33. (Frutas do Brasil, 38).

MANZONI, C.G.; GRÜTZMACHER, A.D.; GIOLO, F.P.; HÄRTER, W.R.; MÜLLER, C. Seletividade de agrotóxicos usados na produção integrada de maçã a adultos de Trichograma pretiosum. Pesquisa Agropecuária Brasileira, Brasilia, v.41, p.1461-1467, 2006a.

MANZONI, C.G.; GRÜTZMACHER, A.D.; GIOLO, F.P.; LIMA, C.A.B.; NÖRNBERG, S.D.; HÄRTER, W.R.;

MÜLLER, C. Seletividade de agrotóxicos recomendados na produção integrada da maçã a Trichogramma pretiosum Riley, 1879 (Hymenoptera:

Trichogrammatidae) em condições de laboratório.

Revista Brasileira de Fruticultura, Jaboticabal, v.28, p.54-257, 2006b.

MANZONI, C.G.; GRÜTZMACHER, A.D.; GIOLO, F.P.; LIMA, C.A.B.; NÖRNBERG, S.D.; MÜLLER, C.;

HÄRTER, W.R. Susceptibilidade de adultos de Trichogramma pretiosum Riley (Hymenoptera: Trichogrammatidae) a fungicidas utilizados no controle de doenças da macieira. Neotropical Entomology, Londrina, v.35, p.223-230, 2006c.

MONTEIRO, L.B.; SOUZA, A. Estratégias integradas para o controle de pragas. In: ENCONTRO NACIONAL SOBRE FRUTICULTURA DE CLIMA TEMPERADO, 7 ., 2004, Fraiburgo. Anais... Caçador: EPAGRI, 2004. p.221225. 
MONTEIRO, L.B.; SOUZA, A.; BELLI, E.L.; SILVA, R.B.Q.; ZUCCHI, R.A. Ocorrência de Trichogramma pretiosum Riley, 1879 (Hymenoptera: Trichogrammatidae) em ovos de Bonagota cranaodes (Meyrick) (Lepidoptera: Tortricidae) em macieira. Revista Brasileira de Fruticultura, Jaboticabal, v.26, p.171-172, 2004.

OMOTO, C. Modo de ação dos inseticidas e resistência de insetos a inseticidas. In: GUEDES, J.C.; COSTA, I.D.; CASTIGLIONI, E. (Eds.). Bases e técnicas de manejo de insetos. Santa Maria: UFSM, 2000. p.31-49.

PINTO, J.D.; KOOPMANSCHAP, A.B.; PLATNER, G.R.; STOUTHAMER, R. The North American Trichogramma (Hymenoptera: Trichogrammatidae) parasitizing certain Tortricidae (Lepidoptera) on apple and pear, with ITS2 DNA characterizations and description of a new species. Biological Control, San Diego, v.23, p.134-142, 2002.

\section{SAS INSTITUTE. Getting started with the SAS learning edition. Cary, 2002.}

SILVA, C.M.S.; FAY, E.F. Agrotóxicos e ambiente.

Brasília: Embrapa Informação Tecnológica, 2004. 400p.

WILLS, G.H.; MCDOWELL, L.L. Pesticide persistence on foliage. Reviews of Environmental Contamination and Toxicology, Summerfield, v.100, p.23-73, 1987.

YOUSSEF, A.I.; NASR, F.N.; STEFANOS, S.S.;

ELKHAIR, S.S.A.; SHEHATA, W.A.; AGAMY, E.; HERZ, A.; HASSAN, S.A. The side-effects of plant protection products used in olive cultivation on the hymenopterous egg parasitoid Trichogramma cacoeciae Marchal. Journal of Applied Entomology, Berlin, v.128, p.593-599, 2004. 